\title{
1D/2D porosity model for urban flood modeling: case of a dense street networks
}

\author{
Pascal Finaud-Guyot ${ }^{1, *}$, Pierre-André Garambois ${ }^{2}$, Shangzhi Chen $^{2}$, Guilhem Dellinger ${ }^{1}$, \\ Abdellah Ghenaim $^{2}$, and Abdellali Terfous ${ }^{2}$ \\ ${ }^{1}$ ICube laboratory (UMR 7357), fluid mechanics team, ENGEES, 1 quai Koch, 67000 Strasbourg, \\ France \\ ${ }^{2}$ ICube laboratory (UMR 7357), fluid mechanics team, INSA Strasbourg, 24 boulevard de la victoire, \\ 67084 Strasbourg cedex, France
}

\begin{abstract}
An increasing urbanization of floodplains has led to higher vulnerability of urban areas and building a new generation of robust, accurate and computationally affordable models dedicated to urban floods is highly required for improving prediction systems and mitigation measures. A better understanding of urban floods hydrodynamics may also be required. In view to achieve computationally affordable and reliable simulations a new $1 \mathrm{D} / 2 \mathrm{D}$ parsimonious hydraulic model Flood1D2D is introduced for flood modeling in complex branched urban networks. It takes advantage of a cut-cell technique and a new effective model able to take into account some essential sub-grid flow features such as flow vein contractions due to recirculations downstream of a crossroad. It is shown that this local phenomenon can be correctly reproduced thanks to this $1 \mathrm{D} / 2 \mathrm{D}$ coupled $\mathrm{SW}$ model parameterized with roughness and porosity. The ability of the model to reproduce realistic flood discharge distributions at the subdistrict scale is also tested on a part of the branched network of ICUBE experimental rig. Further studies should tackle the issues of calibration and unsteady modeling.
\end{abstract}

\section{Introduction}

An increasing urbanization of floodplains has led to higher vulnerability of urban areas with various amounts of damages and human losses depending on flood features. Therefore, building a new generation of robust, accurate and computationally affordable models dedicated to urban floods is highly required for improving prediction systems and mitigation measures.

In the field of urban flood modeling several approaches have been proposed using shallow water (SW) equations either in 1D or 2D parameterized with a classical friction law [1], SWE modified with macro scale porosity [2, 3], or even 3D Navier Stokes equations [4].

Each of these hydrodynamic models are more or less adapted in reproducing local and/or global scale free surface flow variables (water flux, depth or cross sectional area) depending on the real physical complexity of the flow. The most complex models are computationally expensive and require a generally unaffordable amount of data to be constrained. For instance, the flow complexity at a crossroad and downstream is at least 2D in space [5,6]. Classical

\footnotetext{
*e-mail: pascal.finaudguyot@engees.unistra.fr
} 
porosity approaches, are able to account the effect of sub-grid building geometry and stagnant waters, reveal to be a good compromise between physical realism of modeled flood flows and computational cost at the district and city scales [3].

In view to achieve computationally affordable and reliable flood simulations in complex branched urban networks, a 1D/2D parsimonious hydraulic model with pororsity Flood1D2D is introduced [7]. This modeling paradigm and parameterization takes advantage of empirical findings on the effects of 3D flow structures on local and global flow patterns in urban geometries, but also of a parsimonious and computationally affordable 1D/2D SW coupling between 1D streets and 2D crossroads achieved with a split cell technique. In the streets, a complementary parameterization of the SW equations with a porosity approach is introduced to account for the effects of recirculation areas occurring downstream of each crossroad.

The conceptual and discrete model are presented and validated in the next section. A discussion around a global scale sensitivity analysis is proposed in section 3. Concluding remarks and perspectives are provided in section 4.

\section{Flood1D2D - An effective model for urban floods}

A new hydrodynamic model has been designed for an effective representation of urban flood flows throughout branched networks of streets and crossroads. It takes advantage of a cutcell technique and introduces a parsimonious effective model able to take into account some essential sub-grid flow features.

\subsection{Conceptual model}

Urban flood flows may involve complex 3D hydrodynamic structures such as those involved at confluences/defluences [8,9] or recirculation zones downstream of crossroads [6]. Such local flow structures may have impact on water repartition, hence on larger scale flow patterns [10]. A 2D SW modeling approach is able to simulate realistic global flow patterns as shown by [4] on the ICUBE urban flood configuration.

The proposed effective modeling approach is based on a full 2D SW model at crossroads and a much cheaper 1D effective approach in streets remaining physically coherent as shown in this paper and sufficiently informative for operational purpose. The modeling paradigm, based on SW equations and illustrated in figure [fig:Effective-1D], consists in : 2D cells in the crossroad and effective 1D cells including the full street width but without constrain in the velocity direction.

Recirculation zones often observed downstream of flow junctions are assumed to be only present in effective 1D cells. As highlighted in section 2.3.2, classical SW equations in 1D are unable to account for recirculation effects on flow variables. Therefore an effective approach is introduced based on the idea of an equivalent flow vein narrowed by the recirculation zone downstream of a crossroad.

This study considers steady-state hydrodynamics and a recirculation area is defined as a zone on which there is on average no flux in the street direction. 1D cells are defined on the whole street width (figure 1) and can contain 2 domains representing:

- $\Omega_{u}$ an ineffective flow area located in a recirculation zone for which the modeled velocity vector is considered as nil.

- $\Omega_{v}$ an effective flow vein.

Flow vein contraction is measured with the variable $\phi=L_{v} / L$ with $L=L_{u}+L_{v}$ where $L$ represents the full width of the $1 \mathrm{D}$ cell and $L_{u}$ (resp. $L_{v}$ ) is the width of the recirculation 
(resp. flow vein). Notice that such a definition of $\phi$, corresponding to the effective flow width with respect to the full cell width, is similar to a porosity as it can be defined in [11].

In both cells, the hydrodynamics is modeled using the full 2D SW equations (1) to make easier the coupling between streets and crossroads [12]:

$$
\frac{\partial}{\partial t}\left[\begin{array}{l}
h \\
q \\
r
\end{array}\right]+\frac{\partial}{\partial x}\left[\begin{array}{c}
q \\
\frac{q^{2}}{h}+\frac{1}{2} g h^{2} \\
\frac{q}{h}
\end{array}\right]+\frac{\partial}{\partial y}\left[\begin{array}{c}
r \\
\frac{q r}{h} \\
\frac{r^{2}}{h}+\frac{1}{2} g h^{2}
\end{array}\right]=\left[\begin{array}{c}
0 \\
g h\left(S_{0, x}-S_{f, x}\right) \\
g h\left(S_{0, y}-S_{f, y}\right)
\end{array}\right]
$$

where $t$ (resp. $x$ and $y$ ) is the time (resp. space), $g$ the gravitational acceleration, $h$ represents the water depth, $q$ (resp. $r$ ) the unit-discharge in the $x$ (resp. $y$ ) direction, $S_{0, x}$ (resp. $S_{0, y}$ ) the bottom slope in the $x$ (resp. $y$ ) direction and $S_{f, x}$ (resp. $S_{f, y}$ ) the friction slope in the $x$ (resp. $y$ ) direction estimated using the classical Strickler formulation:

$$
S_{0, x}=-\frac{\partial z_{b}}{\partial x} \quad S_{0, y}=-\frac{\partial z_{b}}{\partial y} \quad S_{f, x}=-g h \frac{\left(u^{2}+v^{2}\right)^{1 / 2}}{K^{2} h^{4 / 3}} u \quad S_{f, y}=-g h \frac{\left(u^{2}+v^{2}\right)^{1 / 2}}{K^{2} h^{4 / 3}} v
$$

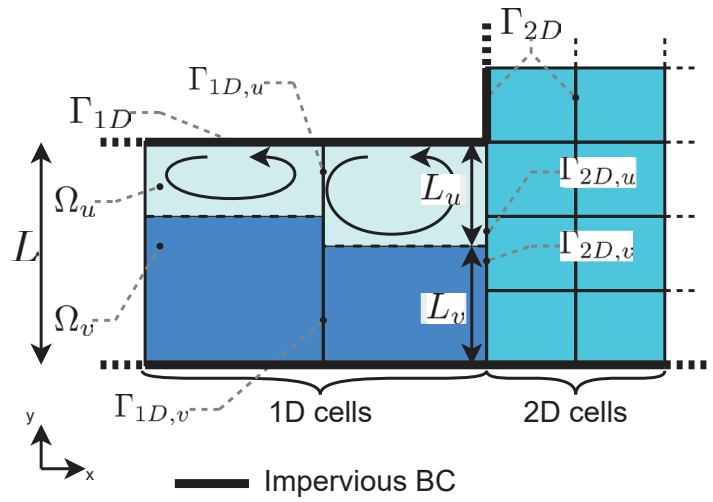

Figure 1. Sketch of a typical mesh configuration describing a 1D/2D "frontal" coupling close to a crossroad/flow junction.

\subsection{Finite volume approach}

On both $1 \mathrm{D}$ or 2D domains, (1) is discretized and solved using a finite volume approach:

$$
\mathbf{U}_{i}^{n+1}=\mathbf{U}_{i}^{n}-\Delta t\left[\frac{1}{A_{i}} \sum_{j \in N(i)} \mathbf{P}_{i, j} L_{j} \mathbf{F}_{j}^{n}+\mathbf{S}\left(\mathbf{U}_{i}^{n}\right)\right]
$$

where $\mathbf{U}_{i}$ is the vector of the modeled variables $\left[\begin{array}{lll}h & q & r\end{array}\right]^{T}, \mathbf{F}_{j}^{n}$ stands for the Riemann fluxes through the interface $j, \mathbf{S}\left(\mathbf{U}_{i}^{n}\right)$ is the source terms vector that applies on cell $i, L_{j}$ is the length of interface $j$ and $\mathbf{P}_{i, j}$ is the matrix that accounts for the coordinate change from the global coordinate system to the one attached to the interface $j$ of cell $i$.

The flux $\mathbf{F}_{j}^{n}$ is estimated using a Riemann solver [2] and thus depends on the left and right states of each interface. 
To take into account the sub-grid flow features (due to recirculation), the sum of the interfaces is subdivided to make appears two elementary fluxes: $F_{u}$ (resp. $F_{v}$ ) through $L_{u, j}$ (resp. $\left.L_{v, j}\right) . L_{u, j}$ is the recirculation width at the interface and is estimated as $L_{u, j}=\left(L_{u, L}+\right.$ $\left.L_{u, R}\right) / 2$ where the subscript ${ }_{L}$ (resp. ${ }_{R}$ ) stands for the cell on the left (resp. right) side of the interface. The definition of $\phi$ allows writing $L_{u}=(1-\phi) \times L_{j}$ leading to:

$$
L_{u, j}=\frac{\left(L_{u, L}+L_{u, R}\right)}{2}=\left[1-\frac{\phi_{L}+\phi_{R}}{2}\right] \times L_{j} \quad L_{v, j}=\frac{\phi_{L}+\phi_{R}}{2} \times L_{j}
$$

With such considerations, (3) can be written either for the $1 \mathrm{D}$ or the $2 \mathrm{D}$ cells as :

$$
\mathbf{U}_{\{1 D, 2 D\}, i}^{n+1}=\mathbf{U}_{\{1 D, 2 D\}, i}^{n}-\Delta t\left[\frac{1}{A_{i}} \sum_{j \in \Gamma_{\{1 D, 2 D\}, v}} \mathbf{P}_{i, j} L_{v, j} \mathbf{F}_{v, j}^{n}+\sum_{j \in \Gamma_{\{1 D, 2 D\}}} \mathbf{P}_{i, j} L_{j} \mathbf{F}_{j}^{n}+\sum_{j \in \Gamma_{\{1 D, 2 D\}, u}} \mathbf{P}_{i, j} L_{u, j} \mathbf{F}_{u, j}^{n}+\mathbf{S}\left(\mathbf{U}_{i}^{n}\right)\right]
$$

In the numerical tool Flood1D2D, the fluxes are computed using the PorAS Riemann solver [2] based on $\mathbf{U}_{L}$ and $\mathbf{U}_{R}$, the hydraulic variables in the left-hand and right-hand cells of the interface. $\mathbf{U}$ being computed as $\left[\begin{array}{lll}h & q / \phi & r / \phi\end{array}\right]^{T}$ (resp. $\left[\begin{array}{lll}h & 0 & 0\end{array}\right]^{T}$ ) when computing through $L_{v}\left(\operatorname{resp} . L_{u}\right)$.

\subsection{Validation}

The proposed model is validated here on a dambreak experiment in a bend and a four branches crossroad with porosity. A detailed validation of Flood1D2D is presented [7], including a comparison to analytical configurations (with and without porosity) and with the classical 2D shallow water model Telemac.

\subsubsection{Dambreak in a $90^{\circ}$ bend}

This first test case aims to highlight that this "frontal" 1D/2D coupling without porosity allows reproducing satisfactorily a highly transient dambreak experiment in open channel with $90^{\circ}$ bend [13].

The experimental geometry is composed of a rectangular channel of width $B=0.495 \mathrm{~m}$ with a $90^{\circ}$ bend connected to an upstream reservoir and ended with a free outlet. The precise dimensions of the domain are provided in [13]. The channel is initially empty and the initial water level in the reservoir is $z_{\text {ini }}=0.25 \mathrm{~m}$ above the bottom of the channel. At $t=0 \mathrm{~s}$, the water contained in the reservoir is instantaneously released in the channel. The computational domain is meshed using a $1 \mathrm{D} / 2 \mathrm{D}$ structured mesh (see figure 2, top left). A simulation is also performed with Flood1D2D using only a classical 2D modeling of the domain with a structured mesh and the PorAS Riemann solver. In both case, the discretization step is set to $\Delta x=0.025 \mathrm{~m}$.

The computed water depth using Flood1D2D are plotted and compared to the experimental results at various locations and in time (figure 2). Using both approaches (1D2D or 2D), the water depth map highlights the mobile hydraulic jump generated by the bend and traveling backward to the reservoir; very similar results can be observed in [13]. The computed water depth profiles are fairly close to the experimental ones. The water depth increases abruptly two times: first when the water flows from the reservoir and then when the hydraulic jump moves backward in the upstream part of the channel rand eaches the gauging points G4 to G1. The farther the point form the reservoir the shorter is the interval between those two increases of $h$. Such evolution do not appears for the last gauging point (G11 - cyan curve) as it is located downstream of the bend. After the second peak, the water depth decreases 


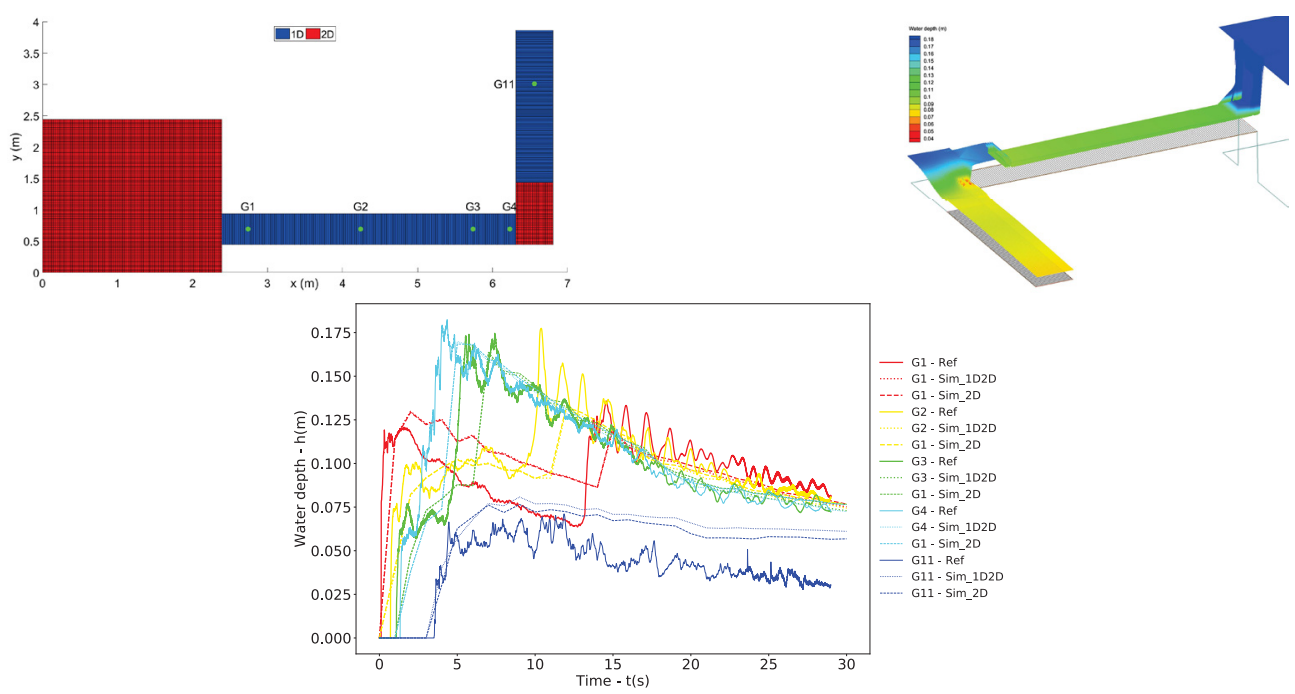

Figure 2. Experimental dambreak in a bend. Top left - 1D/2D mesh; top right - Simulated water surface at $t=7 \mathrm{~s}$; bottom - comparison of the temporal evolution of the water depth between experiment (plain line) and simulation with 1D2D model (dot line) and classical 2D model (dashed line)

slowly for every gauging point, simulation being in fairly good agreement. Note that water depth in the downstream channel is overestimated by the model (G11 - cyan curve), which can be explained by the boundary condition specified as a free outlet. It is coherent with a free fall for supercritical flow but not for subcritical flow as the water depth at the fall is then the critical depth.

\subsubsection{Four branches crossroad}

Flood1D2D outputs with and without porosity are compared to Telemac2D ones with a $k-\epsilon$ model chosen as reference on a 4 branches crossroad in this section. The recirculation shape hence the porosity value of each $1 \mathrm{D}$ cell of Flood1D2D is defined from an empirical equation from [5].

Flood1D2D water depth profiles along the West-East street are presented on figure 3 along with $2 \mathrm{D}$ reference water depth plotted on line $\mathrm{AB}$. Water depth profiles in the street upstream of the crossroad are comparable to the reference with a difference on the order of $5 \mathrm{~mm}$ (for an averaged water depth of $0.11 \mathrm{~m}$ ). Downstream of the crossroad, because of a recirculation the flow vein is contracted, hence flow is accelerated and its free surface presents an inflection for $x \in[3.5 ; 5]$. This shape is correctly reproduced by Flood1D2D parameterized with friction and porosity but not with friction only for which water depth steadily decreases from upstream to downstream.

Even with a distributed friction coefficient such contracted flow profiles cannot be reproduced using a 1D approach as the friction coefficient is embedded in the source term $S_{f}(2)$ of the SW equation. Indeed this term is of the opposite sign of the velocity which remains monotonous in a prismatic reach as water depth profile.

The proposed model uses an effective 1D approach with a porosity able to account for flow contraction due to a recirculation and simulate a realistic water depth profile. A calibration of the predicted porosity shape and the roughness would help to fit more closely Flood1D2D results to the reference. 
As a matter of facts, the proposed approach is able to reproduce a realistic contracted flow pattern downstream of a crossroad by introducing a parameterization using a porosity function $\phi$. Section 3 discusses the effects of this parameterization at a larger scale.
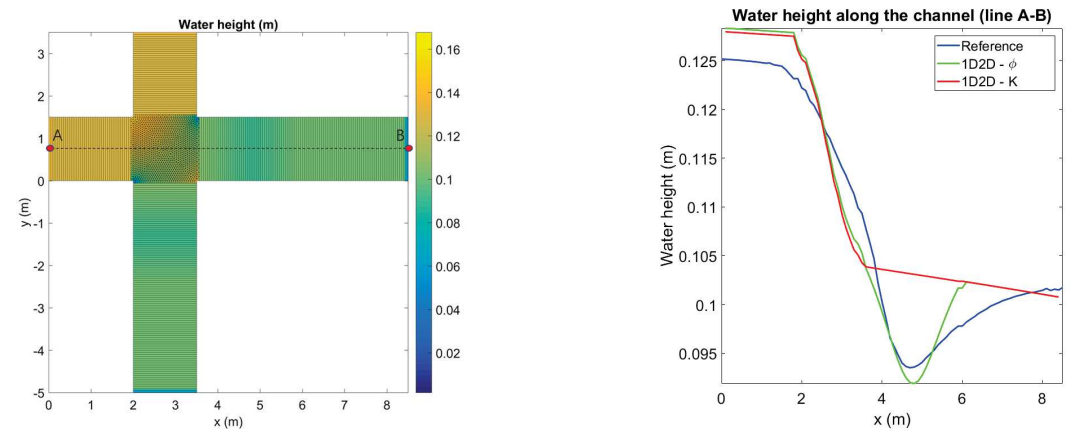

Figure 3. Four branches crossroad modeling. Left: Map of the steady state water depth simulated using the proposed Flood1D2D model with $K$ and $\phi$; equal inflow discharge $Q=0.075 \mathrm{~m}^{3} . \mathrm{s}^{-1}$ in the north and west streets, rating curve as a the downstream boundary condition on west and south streets. Right: water depth profile using various modeling approaches.

\section{Discussion: Flood1D2D response surface}

As illustrated in section 2.3.2 : adding the new $\phi$ parameterization allows reproducing, at the local scale, realistic contracted flow profiles whereas $K$ alone not. Does improving locally these simulated flow features improves global scale hydrodynamics forecasts? The sensitivity of Flood1D2D is thus compared on a crossroads network (see section 3.1) with two different parameterizations: a first one with no porosity and a spatially varied Strickler coefficient and a second one with a uniform Strickler coefficient and a spatially varied porosity in each street.

\subsection{Modeled crossroads network}

The sensitivity of Flood1D2D is determined on a sub-part of the 'ICUBE Urban flood experimental rig' developped in Strasbourg [10].

The sub-part chosen includes four crossroads with streets of various widths and orientations. A set of experimental data allows knowing the discharge and the water depth patterns in each street.

The network is modeled using a $1 \mathrm{D} / 2 \mathrm{D}$ structured mesh with a discretization step $\Delta x=$ $0.025 \mathrm{~m}$ (see figure 4). The boundary conditions are set to upstream discharge in streets $\mathrm{E}$, F, 3 and 4 (corresponding to the experimental values) and rating curves computed for each downstream end. In every configuration, the simulation is run during 1000 s to ensure steady state flow over the whole domain.

\subsection{4 .1 - SW Characterization in $K_{\text {street }} / K_{\text {cross }}$}

The model is parameterized using two uniform values of the Strickler coefficient: $K_{\text {street }}$ in streets and $K_{\text {cross }}$ in crossroads. Each parameter is taken in the set of value $[25,50,75,100,125,150]$ and all combinations are tested. The street outlet discharge is 

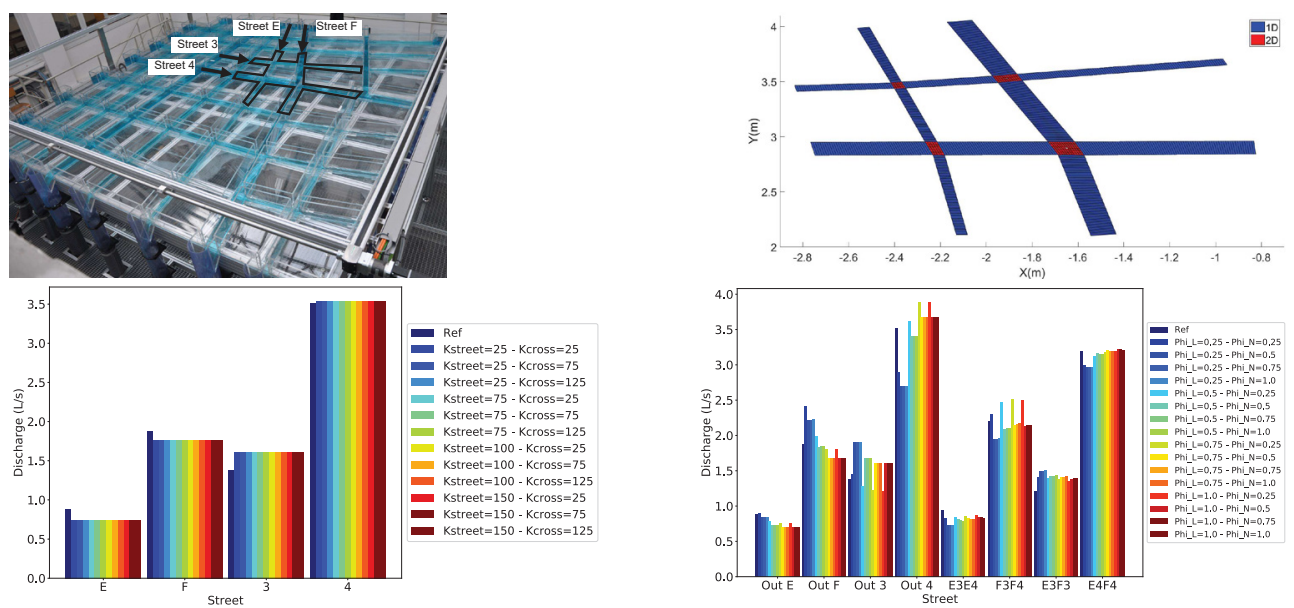

Figure 4. Crossroad network sensitivity analysis. Top left: experimental rig and illustration of the modeled zone; top right: 1D/2D mesh; bottom left: outlet discharge sensitivity to a parameterization with $K_{\text {street }}$ and $K_{\text {cross }}$; bottom right: outlet and street discharge sensitivity to a parameterization with $\phi_{r, n}$ and $\phi_{r, l}$. For the sake of clarity, only some simulation results have been plotted.

compared to the experimental value on figure 4. For each street, the first bar corresponds to the experimental value. Whatever the parameterization of the model, each discharge flowing out from streets E and F (resp. 3 and 4) is systematically under- (resp. over-) estimated. Interestingly, $K_{\text {street }}$ seems to impose an averaged street discharge value (look for instance the shift in the outlet discharge for street $\mathrm{F}$ when $K_{\text {street }}$ varies from 25 to 50 ). $K_{\text {cross }}$ has a smaller impact on outflow discharge repartition (with always the same trend around the value imposed by $\left.K_{\text {street }}\right)$.

\subsection{SW Parameterization in $K_{\text {unif }} / \phi_{\text {large }} / \phi_{\text {narrow }}$}

The model is parameterized using a uniform value of the roughness coefficient $K=$ $75 \mathrm{~m}^{1 / 3} \cdot \mathrm{s}^{-1}$ since few sensitivity to $K_{\text {cross }}$ is found in the previous spatialization. A distributed porosity is defined for all streets downstream of crossroads following a parabolic profile:

$$
\phi(s)=\left\{\begin{array}{l}
a s^{2}+b s+c \text { for } 0 \leq s \leq 0.5 \quad \text { with } \phi(0)=\phi(1 / 2)=1 \quad \text { and } \quad \phi(1 / 4)=\phi_{r} \\
1 \text { elsewhere }
\end{array}\right.
$$

where $s$ is the adimensional curvilinear abscissa (0 (resp. 1) at the upstream (resp. downstream) end of each street).

The model is parameterized, by adjusting $a, b$ and $c$, with two values of $\phi_{r}: \phi_{r}, l$ in large streets (F and 4$)$ and $\phi_{r}, n$ in narrow streets (E and 3 ). In order to sample Flood ID2D response surface, all the combinations of values of $\phi_{r}, l$ and $\phi_{r}, n$ in $[0.25,0.50,0.75,1.00]$ are tested.

The outlet street discharges and those in the four streets between crossroads are compared to the experimental values on figure 4 . For each street, the first bar corresponds to the experimental value.

For each street and all parameter sets tested, there are both under and over estimations of street outlet discharge which was not possible with roughness only. Despite the sparse sampling of this wide parameter space, the spread of model response quite centered around 
discharge measurements suggests that there may exist parameter sets reproducing those values. It clearly shows the ability of the proposed model and parameterization to model various non monotonous flow patterns and potentially realistic local discharge and global discharge distributions. The issues related to the calibration of spatially distributed $K$ and $\phi$ parameters should be further studied, especially equifinality problems in the light of their influence on discharge repartition at large scale in branched networks.

\section{Conclusion}

This study introduced a new 1D/2D SW model dedicated to effective and computationally affordable urban floods modeling, in view for example of operational use. Its structure and parsimonious parameterization is based on empirical observations and aims at reproducing effectively local and global flow physics.

The proposed Flood1D2D model is successfully validated on a complex unsteady dambreak case in a channel including a sharp bend and also on a four branch crossroad including porosity. It is shown that a classical 1D/2D SW model parameterized with roughness only fails to account for free surface deformations due to flow vein contraction downstream of a crossroad. The ability of the new model to reproduce realistic discharge distributions at larger scale is also tested on a part of the branched network of ICUBE experimental rig. Further studies should tackle:

- The problem of calibrating spatially distributed $K$ and $\phi$ parameters along with their influence on simulated flow variables at the (local) street and crossroad scales and on the discharge repartition at the district and city scales

- The benefit of such a parsimonious parameterization in reducing parametric equifinality problems

- Unsteady parameterizations in order to extend model applicability to very transient flooding events.

\section{References}

[1] P. Finaud-Guyot, C. Delenne, V. Guinot, C. Llovel, Comptes Rendus Mécanique 339, $226(2011)$

[2] P. Finaud-Guyot, C. Delenne, J. Lhomme, V. Guinot, C. Llovel, International Journal for Numerical Methods in Fluids 62, 1299 (2010)

[3] V. Guinot, Advances in Water Resources 37, 40 (2012)

[4] Q. Araud, phdthesis, Université de Strasbourg (2012)

[5] J.L. Best, I. Reid, Journal of Hydraulic Engineering 110, 1588 (1984)

[6] E. Mignot, N. Rivière, R.J. Perkins, A. Paquier, Journal of Hydraulic Engineering 134, $701(2008)$

[7] S. Chen, Ph.D. thesis, Université de Strasbourg (2018)

[8] E.H. Taylor, Transactions of the American Society of Civil Engineers 109, 893 (1944)

[9] N. Rivière, G. Travin, R.J. Perkins, Water Resour. Res. 47, W10517 (2011)

[10] P. Finaud-Guyot, P.A. Garambois, Q. Araud, F. Lawniczak, P. François, J. Vazquez, R. Mosé, Urban Water Journal (2018)

[11] J. Lhomme, Ph.D. thesis, Montpellier 2 university (2006)

[12] P. Finaud-Guyot, phdthesis, Université Montpellier II - Sciences et Techniques du Languedoc (2009)

[13] S. Soares-Frazao, Y. Zech, Journal of Hydraulic Engineering 128(11), 956 (2002) 